\title{
EXTENSIONS OF WITNESS MAPPINGS
}

\author{
GEJZA JENČA
}

\begin{abstract}
We deal with the problem of coexistence in interval effect algebras using the notion of a witness mapping. Suppose that we are given an interval effect algebra $E$, a coexistent subset $S$ of $E$, a witness mapping $\beta$ for $S$, and an element $t \in E \backslash S$. We study the question whether there is a witness mapping $\beta_{t}$ for $S \cup\{t\}$ such that $\beta_{t}$ is an extension of $\beta$. In the main result, we prove that such an extension exists if and only if there is a mapping $e_{t}$ from finite subsets of $S$ to $E$ satisfying certain conditions. The main result is then applied several times to prove claims of the type "If $t$ has a such-and-such relationship to $S$ and $\beta$, then $\beta_{t}$ exists".
\end{abstract}

\section{INTRODUCTION AND MOTIVATION}

Let $E$ be an effect algebra. We say that a subset $S$ of $E$ is coexistent if and only if there is a Boolean elgebra $B$ and a morphism of effect algebras $\phi: B \rightarrow E$ (an observable) such that $S$ is a subset of the range of $E$.

Every orthomodular lattice is an effect algebra. It is obvious that a subset of an orthomodular lattice is coexistent if and only if it is a subset of a block. This fact has a nice generalization for lattice-ordered effect algebras: a subset of a lattice ordered effect algebra is coexistent if and only if it is a subset of a maximal MV-subalgebra [17, 11.

From the point of view of physics, the notion of coexistence is well motivated by its application in mathematical foundations of quantum mechanics, see for example [15] and [3]. From the purely mathematical point of view, one can hope that the study of coexistent subsets can shed at least some light at the enigmatic structure of general effect algebras.

To deal with the notion of coexistence, in [10 we introduced and studied a new notion called witness mapping. For a subset $S$ of an interval effect algebra $E$ in a partially ordered abelian group $G$, a witness mapping for $S$ is a mapping from the finite subsets of $S$ to $E$ satisfying certain conditions.

The most important result about witness mappings is the following theorem.

Theorem 1. (Theorem 3 of [10]) Let $E$ be an interval effect algebra. $S \subseteq E$ admits a witness mapping if and only if $S$ is coexistent.

That means that the existence of a witness mapping for $S$ is equivalent to the existence of an observable such that $S$ is a subset of its range. However, the definition of a witness mappings is given purely in terms of interval effect algebras. Moreover, despite of the fact that the proof of Theorem 1 in [10 is constructive

1991 Mathematics Subject Classification. Primary: 03G12, Secondary: 06F20, 81P10.

Key words and phrases. effect algebra, coexistent observables, witness mappings.

This research is supported by grant VEGA G-1/0080/10 of MŠ SR, Slovakia and by the Slovak Research and Development Agency under the contract No. APVV-0071-06. 
in both directions, the relationship between observables and witness maps is far from an one-to-one correspondence: in general, an observable gives a rise to many different witness maps (see the proof of Proposition 6 of [10 in the context of Corollaries 3 and 4 of that paper).

These facts give us the hope that using witness mappings we can deal with questions concerning coexistence without explicitly dealing with observables.

In the present paper, we continue our study of witness maps in a natural direction. Suppose that we are given an interval effect algebra $E$, a coexistent subset $S$ of $E$ a witness mapping $\beta: \operatorname{Fin}(S) \rightarrow E$ and an element $t \in E \backslash S$. We want to know whether is it possible to find a witness mapping $\beta_{t}: \operatorname{Fin}(S \cup\{t\}) \rightarrow E$ such that $\beta_{t}$ is an extension of $\beta$. The question of the existence of such an extension is settled by Theorem 3. We prove that $\beta_{t}$ exists if and only if there is a mapping $e_{t}: \operatorname{Fin}(S) \rightarrow E$ with a certain relationship to $\beta$. In the remaining part of this paper, Theorem 3 is applied several times to prove claims of the type "If $t$ has a such-and-such relationship to $S$ and $\beta$, then $\beta_{t}$ exists".

\section{Definitions AND BASIC RELATIONSHiPS}

2.1. Effect algebras. An effect algebra is a partial algebra $(E ; \oplus, 0,1)$ with a binary partial operation $\oplus$ and two nullary operations 0,1 satisfying the following conditions.

(E1) If $a \oplus b$ is defined, then $b \oplus a$ is defined and $a \oplus b=b \oplus a$.

(E2) If $a \oplus b$ and $(a \oplus b) \oplus c$ are defined, then $b \oplus c$ and $a \oplus(b \oplus c)$ are defined and $(a \oplus b) \oplus c=a \oplus(b \oplus c)$.

(E3) For every $a \in E$ there is a unique $a^{\prime} \in E$ such that $a \oplus a^{\prime}$ exists and $a \oplus a^{\prime}=1$.

(E4) If $a \oplus 1$ is defined, then $a=0$.

Effect algebras were introduced by Foulis and Bennett in their paper [7].

In their paper [14, Chovanec and Kôpka introduced an essentially equivalent structure called $D$-poset. Their definition is an abstract algebraic version the $D$ poset of fuzzy sets, introduced by Kôpka in the paper [13].

Another equivalent structure was introduced by Giuntini and Greuling in 9 . We refer to [6] for more information on effect algebras and related topics.

2.2. Properties of effect algebras. In an effect algebra $E$, we write $a \leq b$ if and only if there is $c \in E$ such that $a \oplus c=b$. It is easy to check that for every effect algebra $E, \leq$ is a partial order on $E$. Moreover, it is possible to introduce a new partial operation $\ominus ; b \ominus a$ is defined if and only if $a \leq b$ and then $a \oplus(b \ominus a)=b$. It can be proved that, in an effect algebra, $a \oplus b$ is defined if and only if $a \leq b^{\prime}$ if and only if $b \leq a^{\prime}$. In an effect algebra, we write $a \perp b$ if and only if $a \oplus b$ exists.

A finite family $\left(a_{1}, \ldots, a_{n}\right)$ of elements of an effect algebra is called orthogonal if and only if the sum $a_{1} \oplus \cdots \oplus a_{n}$ exists. An orthogonal family $\left(a_{1}, \ldots, a_{n}\right)$ is a decomposition of unit if and only if $a_{1} \oplus \ldots a_{n}=1$.

2.3. Classes of effect algebras. The class of effect algebras can be considered a common superclass of several important classes of algebras: orthomodular lattices [12, 2], orthoalgebras [8], MV-algebras [4, 16].

- An effect algebra $E$ is an orthomodular lattice if and only if $E$ is latticeordered and, for all $a, b \in E, a \perp b \Longrightarrow a \wedge b=0$. 
- An effect algebra $E$ is an $M V$-effect algebra if and only if $E$ is lattice-ordered and, for all $a, b \in E, a \ominus(a \wedge b)=(a \vee b) \ominus b$. By [5], there is a natural, one-to-one correspondence between MV-algebras and MV-effect algebras.

- An effect algebra $E$ is a Boolean algebra if and only if $E$ is an orthomodular lattice and $E$ is an MV-effect algebra. In this case, we wave $a \perp b$ iff $a \wedge b=0$ and then $a \oplus b=a \vee b$.

2.4. Observables and coexistent subsets. Let $E, F$ be effect algebras. A mapping $\phi: E \rightarrow F$ is a morphism of effect algebras if and only if the following conditions are satisfied:

(EM1) $\phi(1)=1$.

(EM2) If $a, b \in E, a \perp b$ then $\phi(a) \perp \phi(b)$ and $\phi(a \oplus b)=\phi(a) \oplus \phi(b)$.

We note that every morphism of effect algebras is isotone. Moreover, every morphism of effect algebras preserves the 0 element, as well as the unary operation $x \mapsto x^{\prime}$ and the partial binary operation $\ominus$.

A bijective morphism of effect algebras $\phi: E \rightarrow F$ such that $\phi^{-1}$ is a morphism of effect algebras is called an isomorphism of effect algebras.

Let $B$ be a Boolean algebra and let $E$ be an effect algebra. A morphism of effect algebras $\alpha: B \rightarrow E$ is called an observable. If $B$ is finite, then we say that $\alpha$ is a $a$ simple observable.

Definition 1. We say that a subset $S$ of an effect algebra is coexistent if there exists a Boolean algebra $B$ and an observable $\alpha: B \rightarrow E$ such that $S \subseteq \alpha(B)$.

2.5. Interval effect algebras. Let $(G, \leq)$ be a partially ordered abelian group and $u \in G$ be a positive element. For $0 \leq a, b \leq u$, define $a \oplus b$ if and only if $a+b \leq u$ and put $a \oplus b=a+b$. With such a partial operation $\oplus$, the closed interval

$$
[0, u]_{G}=\{x \in G: 0 \leq x \leq u\}
$$

becomes an effect algebra $\left([0, u]_{G}, \oplus, 0, u\right)$. Effect algebras which arise from partially ordered abelian groups in this way are called interval effect algebras, see [1].

By [16, every MV-effect algebra is an MV-algebra.

2.6. Standard effect algebras. Let $\mathbb{H}$ be a Hilbert space, let $\mathcal{B}_{s a}(\mathbb{H})$ be the set of all bounded self-adjoint operators on $\mathbb{H}$. For $A, B \in \mathcal{B}_{s a}(\mathbb{H})$, write $A \leq B$ if and only if, for all $x \in \mathbb{H},\langle A x, x\rangle \leq\langle B x, x\rangle$. Then $\left(\mathcal{B}_{s a}(\mathbb{H}),+, 0\right)$ is a partially ordered abelian group. The identity operator $I$ is a positive element of this group.

The prototype interval effect algebra is the standard effect algebra $\mathcal{E}(\mathbb{H})=$ $[0, I]_{\mathcal{B}_{s a}(\mathbb{H})} \cdot \mathcal{E}(\mathbb{H})$ plays an important role in the unsharp observable approach to the foundations of quantum mechanics, see for example [3].

2.7. Witness mappings. Let $E$ be an interval effect algebra in a partially ordered abelian group $G$. Let $S \subseteq E$. Let us write $\operatorname{Fin}(S)$ for the set of all finite subsets of $S$. We write $I(\operatorname{Fin}(S))$ for the set of all comparable elements of the poset $(\operatorname{Fin}(S), \subseteq)$, that means,

$$
I(\operatorname{Fin}(S))=\{(X, Y) \in \operatorname{Fin}(S) \times \operatorname{Fin}(S): X \subseteq Y\} .
$$

For every mapping $\beta: \operatorname{Fin}(S) \rightarrow G$, we define a mapping $D_{\beta}: I(\operatorname{Fin}(S)) \rightarrow G$. For $(X, A) \in I(\operatorname{Fin}(S))$, the value $D_{\beta}(X, A) \in G$ is given by the rule

$$
D_{\beta}(X, A):=\sum_{X \subseteq Z \subseteq A}(-1)^{|X|+|Z|} \beta(Z) .
$$


The transform $\beta \mapsto D_{\beta}$ is (essentially) a Möbius inversion with respect to the poset $(\operatorname{Fin}(S), \subseteq)$; see 10 for details. When dealing with $D_{\beta}$, the following lemma is crucial.

Lemma 1. (Lemma 1 of 10 ) Let $E$ be an interval effect algebra in a partially ordered abelian group $G$. Let $S$ be a subset of $E$, let $\beta: \operatorname{Fin}(S) \rightarrow G$. For all $c \in S \backslash A$,

$$
D_{\beta}(X, A)=D_{\beta}(X, A \cup\{c\})+D_{\beta}(X \cup\{c\}, A \cup\{c\}) .
$$

In [10], we introduced and studied the following notion:

Definition 2. Let $E$ be an interval effect algebra. We say that a mapping $\beta$ : $\operatorname{Fin}(S) \rightarrow E$ is a witness mapping for $S$ if and only if the following conditions are satisfied.

(A1) $\beta(\emptyset)=1$,

(A2) for all $c \in S, \beta(\{c\})=c$,

(A3) for all $(X, A) \in I(\operatorname{Fin}(S)), D_{\beta}(X, A) \geq 0$.

The most important result concerning witness mappings is the following theorem.

Theorem 2. (10, Theorem 3) Let $E$ be an interval effect algebra. $S \subseteq E$ admits a witness mapping if and only if $S$ is coexistent.

There are at least two important examples of witness mappings:

Example 1. (Corollary 2 of [10]) Let $M$ be an MV-effect algebra. The mapping $\bigwedge: \operatorname{Fin}(M) \rightarrow M$ is a witness mapping.

Example 2. (Proposition 9 of [10]) Let $S$ be a pairwise commuting subset of $\mathcal{E}(\mathbb{H})$. The mapping $\Pi: \operatorname{Fin}(S) \rightarrow \mathcal{E}(\mathbb{H})$ given by

$$
\Pi\left(\left\{x_{1}, \ldots, x_{n}\right\}\right)=x_{1} \ldots x_{n} .
$$

is a witness mapping.

Several properties of the witness mappings $\Pi$ and $\Lambda$ generalize nicely to all witness mappings, as the following proposition shows.

Proposition 1. (Propositions 3 and 5 of [10])

(a) For all $(X, A) \in I(\operatorname{Fin}(S)), D_{\beta}(X, A) \leq 1$.

(b) $\beta$ is an antitone mapping from $(\operatorname{Fin}(S), \subseteq)$ to $(E, \leq)$.

(c) For all $X \in \operatorname{Fin}(S), \beta(X)$ is a lower bound of $X$.

(d) Suppose that $0 \in S$. If $0 \in X \in \operatorname{Fin}(S)$, then $\beta(X)=0$.

(e) Suppose that $1 \in S$. For all $X \in \operatorname{Fin}(S), \beta(X)=\beta(X \cup\{1\})$

\section{EXTENSIONS OF WITNESS MAPPINGS IN INTERVAL EFFECT ALGEBRAS}

Let $E$ be an interval effect algebra, let $S \subseteq E$ and let $\beta$ be a witness mapping for $S$. We call the pair $(\beta, S)$ a witness pair in $E$. Suppose that there is another witness pair $\left(\beta^{+}, S^{+}\right)$such that $S^{+} \supseteq S$ and $\beta^{+}$restricted to $\operatorname{Fin}(S)$ is equal to $\beta$. We then say that $\left(\beta^{+}, S^{+}\right)$extends $(\beta, S)$, in symbols $\left(\beta^{+}, S^{+}\right) \sqsupseteq(\beta, S)$. If is easy to see that $\sqsupseteq$ is a partial order on the set of all witness pairs. By a standard use of Zorn lemma, it is easy to check that above every witness there is a maximal witness pair. If $t \in E \backslash S$ and there is a witness pair $\left(\beta_{t}, S \cup\{t\}\right)$ that extends $(\beta, S)$, then we say that $(\beta, S)$ can be extended by $t$.

In the remainder of this section, $E$ is an interval effect algebra and $(\beta, S)$ is a witness pair in $E$. 
Theorem 3. Let $t \in E \backslash S$. The following are equivalent:

(a) $(\beta, S)$ can be extended by $t$.

(b) There is a mapping $e_{t}: \operatorname{Fin}(S) \rightarrow E$ such that $e_{t}(\emptyset)=t$ and $0 \leq D_{e_{t}} \leq D_{\beta}$.

Proof. Let us prove that (a) implies (b). Suppose that $(\beta, S)$ can be extended by $t$. Let $\left(\beta_{t}, S \cup\{t\}\right)$ be a witness pair that extends $(\beta, S)$. For $Y \in \operatorname{Fin}(S)$, put $e_{t}(Y)=\beta_{t}(Y \cup\{t\})$. Clearly, $e_{t}(\emptyset)=t$. For all $X, A \in \operatorname{Fin}(S)$ with $X \subseteq A$,

$$
\begin{array}{r}
D_{e_{t}}(X, A)=\sum_{X \subseteq Y \subseteq A}(-1)^{|X|+|Y|} e_{t}(Y)= \\
=\sum_{X \subseteq Y \subseteq A}(-1)^{|X|+|Y|} \beta_{t}(Y \cup\{t\})
\end{array}
$$

A substitution $Z=Y \cup\{t\}$ yields

$$
\begin{array}{r}
\sum_{X \subseteq Y \subseteq A}(-1)^{|X|+|Y|} \beta_{t}(Y \cup\{t\})= \\
=\sum_{X \cup\{t\} \subseteq Z \subseteq A \cup\{t\}}(-1)^{|X|+|Z \cup\{t\}|} \beta_{t}(Z)= \\
=\sum_{X \cup\{t\} \subseteq Z \subseteq A \cup\{t\}}(-1)^{|X \cup\{t\}|+|Z|} \beta_{t}(Z)= \\
=D_{\beta_{t}}(X \cup\{t\}, A \cup\{t\}) \geq 0,
\end{array}
$$

since $\beta_{t}$ is a witness mapping. Therefore, $D_{e_{t}}(X, A) \geq 0$. By Lemma 1 ,

$$
D_{\beta_{t}}(X, A)=D_{\beta_{t}}(X, A \cup\{t\})+D_{\beta_{t}}(X \cup\{t\}, A \cup\{t\}) .
$$

As $\beta_{t}$ is a witness mapping, $D_{\beta_{t}}(X, A \cup\{t\}) \geq 0$. Thus,

$$
D_{e_{t}}(X, A)=D_{\beta_{t}}(X \cup\{t\}, A \cup\{t\}) \leq D_{\beta_{t}}(X, A) .
$$

Let us prove that (b) implies (a). The mapping $\beta_{t}: \operatorname{Fin}(S \cup\{t\}) \rightarrow E$ is given by

$$
\beta_{t}(X)= \begin{cases}\beta(X) & \text { for } t \notin X, \\ e_{t}(X \backslash\{t\}) & \text { for } t \in X .\end{cases}
$$

Obviously, $\beta_{t}(\emptyset) \beta(\emptyset)=1$ and, for all $c \in S \cup\{t\}, \beta_{t}(\{c\})=c$.

It remains to prove that $D_{\beta_{t}} \geq 0$. Let $X, A \in \operatorname{Fin}(S \cup\{t\})$ be such that $X \subseteq A$. (Case 1) $t \notin A$.

If $t \notin A$, then $t \notin X$ and $D_{\beta_{t}}(X, A)=D_{\beta}(X, A) \geq 0$.

(Case 2) $t \in X$.

If $t \in X$, then $t \in A$ and

$$
\begin{aligned}
D_{\beta_{t}}(X, A) & =\sum_{X \subseteq Z \subseteq A}(-1)^{|X|+|Z|} \beta_{t}(Z)= \\
= & \sum_{X \subseteq Z \subseteq A}(-1)^{|X|+|Z|} e_{t}(Z \backslash\{t\})
\end{aligned}
$$


A substitution $Y=Z \backslash\{t\}$ yields

$$
\begin{array}{r}
\sum_{X \subseteq Z \subseteq A}(-1)^{|X|+|Z|} e_{t}(Z \backslash\{t\})= \\
=\sum_{X \backslash\{t\} \subseteq Y \subseteq A \backslash\{t\}}(-1)^{|X|+|Y \cup\{t\}|} e_{t}(Y)= \\
=\sum_{X \backslash\{t\} \subseteq Y \subseteq A \backslash\{t\}}(-1)^{|X \backslash\{t\}|+|Y|} e_{t}(Y)=D_{e_{t}}(X \backslash\{t\}, A \backslash\{t\}) \geq 0,
\end{array}
$$

by assumption.

(Case 3) $t \notin X$ and $t \in A$.

Put $A_{0}=A \backslash\{t\}$ so that $A=A_{0} \cup\{t\}$ and $D_{\beta_{t}}(X, A)=D_{\beta_{t}}\left(X, A_{0} \cup\{t\}\right)$. By Lemma 1

$$
D_{\beta_{t}}\left(X, A_{0}\right)=D_{\beta_{t}}\left(X, A_{0} \cup\{t\}\right)+D_{\beta_{t}}\left(X \cup\{t\}, A_{0} \cup\{t\}\right),
$$

hence

$$
D_{\beta_{t}}\left(X, A_{0} \cup\{t\}\right)=D_{\beta_{t}}\left(X, A_{0}\right)-D_{\beta_{t}}\left(X \cup\{t\}, A_{0} \cup\{t\}\right) .
$$

Since $t \notin X$ and $t \notin A_{0}$, we see that $D_{\beta_{t}}\left(X \cup\{t\}, A_{0} \cup\{t\}\right)=D_{e_{t}}\left(X, A_{0}\right)$ by the proof of (Case 2). Hence,

$D_{\beta_{t}}\left(X, A_{0} \cup\{t\}\right)=D_{\beta_{t}}\left(X, A_{0}\right)-D_{\beta_{t}}\left(X \cup\{t\}, A_{0} \cup\{t\}\right)=D_{\beta_{t}}\left(X, A_{0}\right)-D_{e_{t}}\left(X, A_{0}\right)$.

As $D_{\beta_{t}} \geq D_{e_{t}}$, this implies that $D_{\beta_{t}}\left(X, A_{0} \cup\{t\}\right) \geq 0$. It remains to recall that $D_{\beta_{t}}(X, A)=D_{\beta_{t}}\left(X, A_{0} \cup\{t\}\right)$.

Proposition 2. Every witness pair $(\beta, S)$ can be extended by 0 and 1 .

Proof. Put $e_{0}(X)=0, e_{1}(X)=\beta(X)$ and apply Theorem 3

Proposition 3. Suppose $u \in S, u^{\prime} \notin S$. Then $(\beta, S)$ can be extended by $u^{\prime}$.

Proof. We shall apply Theorem 3, Put

$$
e_{u^{\prime}}(X)=\beta(X)-\beta(X \cup\{u\}) .
$$

We see that

$$
e_{u^{\prime}}(\emptyset)=\beta(\emptyset)-\beta(\{u\})=1-u=u^{\prime} .
$$

Let $X, A \in \operatorname{Fin}(S)$ be such that $X \subseteq A$. It remains to prove that $0 \leq D_{e_{t}}(X, A) \leq$ $D_{\beta}(X, A)$.

(Case 1) $u \in X$.

Obviously, $\beta(Z)=\beta(Z \cup\{u\})$, so $D_{e_{u^{\prime}}}(X, A)=0$.

(Case 2) $u \notin A$

Let us rewrite

$$
\begin{aligned}
D_{e_{u^{\prime}}}(X, A) & =\sum_{X \subseteq Z \subseteq A}(-1)^{|X|+|Z|} \beta(Z)-\beta(Z \cup\{u\})= \\
& =\sum_{X \subseteq Z \subseteq A}(-1)^{|X|+|Z|} \beta(Z)-\sum_{X \subseteq Z \subseteq A}(-1)^{|X|+|Z|} \beta(Z \cup\{u\})= \\
& =D_{\beta}(X, A)-\sum_{X \cup\{u\} \subseteq Y \subseteq A \cup\{u\}}(-1)^{|X|+|Y \backslash\{u\}|} \beta(Y)
\end{aligned}
$$


A substitution $Y=Z \cup\{u\}$ now yields

$$
\begin{aligned}
& D_{\beta}(X, A)-\sum_{X \cup\{u\} \subseteq Y \subseteq A \cup\{u\}}(-1)^{|X|+|Y \backslash\{u\}|} \beta(Y)= \\
= & D_{\beta}(X, A)-\sum_{X \cup\{u\} \subseteq Y \subseteq A \cup\{u\}}(-1)^{|X \cup\{u\}|+|Y|} \beta(Y)= \\
= & D_{\beta}(X, A)-D_{\beta}(X \cup\{u\}, A \cup\{u\})=D_{\beta}(X, A \cup\{u\}) .
\end{aligned}
$$

Therefore, $0 \leq D_{e_{u^{\prime}}}(X, A) \leq D_{\beta}(X, A)$.

(Case 3) $u \in A$ and $u \notin X$.

Let us put $A_{0}=A \backslash\{u\}$. By Lemma 1,

$$
D_{e_{u^{\prime}}}(X, A)=D_{e_{u^{\prime}}}\left(X, A_{0} \cup\{u\}\right)=D_{e_{u^{\prime}}}\left(X, A_{0}\right)-D_{e_{u^{\prime}}}\left(X \cup\{u\}, A_{0} \cup\{u\}\right) .
$$

By (Case 1),

$$
D_{e_{u^{\prime}}}\left(X \cup\{u\}, A_{0} \cup\{u\}\right)=0,
$$

so

$$
D_{e_{u^{\prime}}}(X, A)=D_{e_{u^{\prime}}}\left(X, A_{0}\right)
$$

and, since $u \notin A_{0}$, (Case 3 ) reduces to (Case 2).

Proposition 4. Suppose that $u \in \operatorname{ran}(\beta)$. Then $(\beta, S)$ can be extended by $u$.

Proof. Since $u \in \operatorname{ran}(\beta))$, there is $U \in \operatorname{Fin}(S)$ such that $\beta(U)=u$. For all $Z \in$ $\operatorname{Fin}(S)$, we put

$$
e_{u}(Z)=\beta(Z \cup U)
$$

Clearly, $e_{u}(\emptyset)=\beta(U)=u$. Further,

$$
D_{e_{u}}(X, A)=\sum_{X \subseteq Z \subseteq A}(-1)^{|X|+|Z|} \beta(Z \cup U)
$$

Note that, for every $Z$, there is a unique pair of sets $\left(Z_{1}, Z_{2}\right)$ such that

$$
\begin{aligned}
& Z=Z_{1} \cup Z_{2} \\
& X \backslash U \subseteq Z_{1} \subseteq A \backslash U \\
& X \cap U \subseteq Z_{2} \subseteq A \cap U .
\end{aligned}
$$

In fact, $Z_{1}=Z \backslash U$ and $Z_{2}=Z \cap U$. Therefore, we may express $D_{e_{u}}(X, A)$ as a double sum:

$$
D_{e_{u}}(X, A)=\sum_{X \backslash U \subseteq Z_{1} \subseteq A \backslash U} \sum_{X \cap U \subseteq Z_{2} \subseteq A \cap U}(-1)^{\left|Z_{1}\right|+\left|Z_{2}\right|+|X|} \beta\left(Z_{1} \cup Z_{2} \cup U\right) .
$$

As $Z_{2} \subseteq U$, we obtain

$$
D_{e_{u}}(X, A)=\sum_{X \backslash U \subseteq Z_{1} \subseteq A \backslash U} \sum_{X \cap U \subseteq Z_{2} \subseteq A \cap U}(-1)^{\left|Z_{1}\right|+\left|Z_{2}\right|+|X|} \beta\left(Z_{1} \cup U\right) .
$$

(Case 1) Suppose that $X \cap U=A \cap U$. Then $Z_{2}=X \cap U$ and the inner sum collapses to a single summand, so

$$
D_{e_{u}}(X, A)=\sum_{X \backslash U \subseteq Z_{1} \subseteq A \backslash U}(-1)^{\left|Z_{1}\right|+|X \cap U|+|X|} \beta\left(Z_{1} \cup U\right) .
$$

We can substitute $Y:=Z_{1} \cup U$, so that the sum can be written as

$$
D_{e_{u}}(X, A)=\sum_{X \cup U \subseteq Y \subseteq A \cup U}(-1)^{|Y \backslash U|+|X \cap U|+|X|} \beta(Y) .
$$


By a simple reasoning about parity, it is easy to check that $(-1)^{|X \cup U|+|U|}=$ $(-1)^{|X \cap U|+|X|}$, hence

$$
\begin{aligned}
D_{e_{u}}(X, A) & =\sum_{X \cup U \subseteq Y \subseteq A \cup U}(-1)^{|Y \backslash U|+|X \cap U|+|X|} \beta(Y)= \\
& =\sum_{X \cup U \subseteq Y \subseteq A \cup U}(-1)^{|Y \backslash U|+|X \cup U|+|U|} \beta(Y)= \\
& =\sum_{X \cup U \subseteq Y \subseteq A \cup U}(-1)^{|Y|+|X \cup U|} \beta(Y)=D_{\beta}(X \cup U, A \cup U) .
\end{aligned}
$$

By the Lemma 3 of [10], for any $C \in \operatorname{Fin}(S)$ with $C \cap A=\emptyset$,

$$
\bigoplus_{Y \subseteq C} D_{\beta}(X \cup Y, A \cup C)=D_{\beta}(X, A) .
$$

This implies that $D_{\beta}(X \cup C, A \cup C) \leq D_{\beta}(X, A)$. Putting $C:=U \backslash A$ yields

$$
D_{\beta}(X \cup(U \backslash A), A \cup(U \backslash A)) \leq D_{\beta}(X, A) .
$$

Since $X \cap U=A \cap U, X \cup(U \backslash A)=X \cup U$ and

$$
D_{\beta}(X \cup(U \backslash A), A \cup(U \backslash A))=D_{\beta}(X \cup U, A \cup U) .
$$

Hence,

$$
D_{\beta}(X \cup U, A \cup U) \leq D_{\beta}(X, A) .
$$

Summarizing, we see that

$$
0 \leq D_{\beta}(X \cup U, A \cup U)=D_{e_{u}}(X, A) \leq D_{\beta}(X, A) .
$$

(Case 2) Suppose that $X \cap U \neq A \cap U$. Let us focus onto the inner sum of (1). Pick $c \in(A \cap U) \backslash(X \cap U)$. Consider the systems of sets

$$
\begin{aligned}
& \mathbb{H}_{c}:=\{H:(X \cap U) \cup\{c\} \subseteq H \subseteq A \cup U\} \\
& \mathbb{H}_{c}^{\prime}:=\{H:(X \cap U) \subseteq H \subseteq(A \cup U) \backslash\{c\}\} .
\end{aligned}
$$

Note that, for all $X \cap U \subseteq Z_{2} \subseteq A \cap U, c \in Z_{2}$ iff $Z_{2} \in \mathbb{H}_{c}$ and $c \notin Z_{2}$ iff $Z_{2} \in \mathbb{H}_{c}^{\prime}$. Moreover, $Z_{2} \mapsto Z_{2} \cup\{c\}$ is a bijection from $\mathbb{H}_{c}^{\prime}$ onto $\mathbb{H}_{c}$. Therefore, we may write

$$
\begin{aligned}
& \sum_{X \cap U \subseteq Z_{2} \subseteq A \cap U}(-1)^{\left|Z_{1}\right|+\left|Z_{2}\right|+|X|} \beta\left(Z_{1} \cup U\right)= \\
= & \sum_{Z_{2} \in \mathbb{H}_{c}^{\prime}}(-1)^{\left|Z_{1}\right|+\left|Z_{2}\right|+|X|} \beta\left(Z_{1} \cup U\right)+(-1)^{\left|Z_{1}\right|+\left|Z_{2} \cup\{c\}\right|+|X|} \beta\left(Z_{1} \cup U\right) .
\end{aligned}
$$

However, it is obvious that

$$
(-1)^{\left|Z_{1}\right|+\left|Z_{2}\right|+|X|} \beta\left(Z_{1} \cup U\right)+(-1)^{\left|Z_{1}\right|+\left|Z_{2} \cup\{c\}\right|+|X|} \beta\left(Z_{1} \cup U\right)=0 .
$$

Thus, $D_{e_{u}}(X, A)=0$ for $X \cap U \neq A \cap U$.

Corollary 1. Let $(\beta, S)$ be a maximal witness pair. Then $0,1 \in S, S$ is closed with respect to $x \mapsto x^{\prime}$, and $\beta$ maps $\operatorname{Fin}(S)$ onto $S$.

Proof. By the Propositions 2,3, and 4 . 


\section{EXTENSIONS IN STANDARD EFFECT ALGEBRAS}

Proposition 5. Let $u \in \mathcal{E}(\mathcal{H})$, suppose that $u$ commutes with every element of $\operatorname{ran}(\beta)$. Then $(\beta, S)$ can be extended by $u$.

Proof. Put $e_{u}(X)=u . \beta(X)$. Clearly, $e_{u}(\emptyset)=u$ and

$$
0 \leq u . D_{\beta}(X, A)=D_{e_{u}}(X, A) \leq D_{\beta}(X, A) .
$$

Proposition 6. Let $u, w \in \mathcal{E}(\mathcal{H})$, suppose that $(\beta, S)$ can be extended by both $u$ and $w$. Let $v$ be a convex combination of $u, w$. Then $(\beta, S)$ can be extended by $v$.

Proof. Write $v=\theta u+(1-\theta) w$, where $\theta \in[0,1]_{\mathbb{R}}$. Let $e_{u}$ and $e_{w}$ be extension mappings for $u$ and $w$, respectively. Put

$$
e_{v}=\theta e_{u}+(1-\theta) e_{w}
$$

Clearly, $e_{v}(\emptyset)=v$ and

$$
D_{e_{v}}(X, A)=\theta D_{e_{u}}(X, A)+(1-\theta) D_{e_{w}}(X, A) .
$$

Since $e_{u}$ and $e_{w}$ are extension mappings,

$$
\begin{aligned}
& 0 \leq D_{e_{u}}(X, A) \leq D_{\beta}(X, A) \\
& 0 \leq D_{e_{w}}(X, A) \leq D_{\beta}(X, A)
\end{aligned}
$$

Multiplying (2) by $\theta$, (3) by $(1-\theta)$ and then summing up the inequalities gives us

$$
0 \leq \theta D_{e_{u}}(X, A)+(1-\theta) D_{e_{w}}(X, A) \leq D_{\beta}(X, A) .
$$

\section{REFERENCES}

[1] M.K. Bennett and D.J. Foulis. Interval and scale effect algebras. Advances in Applied Mathematics, 19:200-215, 1997.

[2] L. Beran. Orthomodular Lattices, Algebraic Approach. Kluwer, Dordrecht, 1985.

[3] P. Busch, P. Lahti, and P. Mittelstaedt. The Quantum Theory of Measurement. Springer Verlag, 2nd edition, 1996.

[4] C.C. Chang. Algebraic analysis of many-valued logics. Trans. Amer. Math. Soc., 88:467-490, 1959.

[5] F. Chovanec and F. Kôpka. Boolean D-posets. Tatra Mt. Math. Publ, 10:183-197, 1997.

[6] A. Dvurečenskij and S. Pulmannová. New Trends in Quantum Structures. Kluwer, Dordrecht and Ister Science, Bratislava, 2000.

[7] D.J. Foulis and M.K. Bennett. Effect algebras and unsharp quantum logics. Found. Phys., 24:1325-1346, 1994.

[8] D.J. Foulis and C.H. Randall. Operational quantum statistics. I. Basic concepts. J. Math. Phys., 13:1667-1675, 1972.

[9] R. Giuntini and H. Greuling. Toward a formal language for unsharp properties. Found. Phys., 19:931-945, 1989.

[10] G. Jenča. Coexistence in interval effect algebras. Proceedings of AMS (to appear), arXiv:quant-ph/0910.2823.

[11] G. Jenča. Boolean algebras R-generated by MV-effect algebras. Fuzzy sets and systems, 145:279-285, 2004.

[12] G. Kalmbach. Orthomodular Lattices. Academic Press, New York, 1983.

[13] F. Kôpka. D-posets of fuzzy sets. Tatra Mt. Math. Publ., 1:83-87, 1992.

[14] F. Kôpka and F. Chovanec. D-posets. Math. Slovaca, 44:21-34, 1994.

[15] G. Ludwig. Foundations of Quantum Mechanics. Springer-Verlag, Berlin, 1983.

[16] D. Mundici. Interpretation of AF $C^{*}$-algebras in Lukasziewicz sentential calculus. J. Functional Analysis, 65:15-53, 1986. 
[17] Z. Riečanová. A generalization of blocks for D-lattices and lattice effect algebras. Int. J. Theor. Phys., 39:231-237, 2000.

Department of Mathematics and Descriptive Geometry, Faculty of Civil Engineering, Slovak Technical University, Radlinského 11, Bratislava 813 68, Slovak Republic

E-mail address: gejza.jenca@stuba.sk 